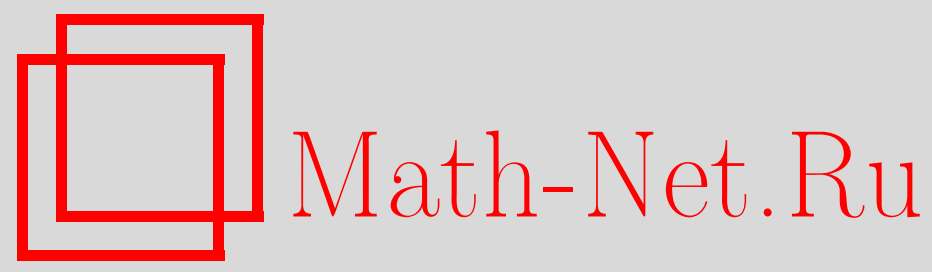

А. Н. Старков, Минимальность и строгая эргодичность однородных действий, Матем. заметки, 1999, том 66, выпуск 2, 293-301

DOI: https://doi.org/10.4213/mzm1167

Использование Общероссийского математического портала Math-Net.Ru подразумевает, что вы прочитали и согласны с пользовательским соглашением http://www . mathnet.ru/rus/agreement

Параметры загрузки:

IP : 3.95 .254 .165

26 апреля 2023 г., 11:13:03






\title{
МИНИМАЛЬНОСТЬ И СТРОГАЯ ЭРГОДИЧНОСТЬ ОДНОРОДНЫХ ДЕЙСТВИЙ
}

\author{
А. Н. Старков
}

Пусть $\Gamma$ - решетка в связной группе Ли $G, H \subset G$ - связная подгруппа и Ad-присоединенное представление $G$ на своей алгебре Ли g. Предположим, что $\operatorname{Ad}(H)$ расщепляется в полупрямое произведение редуктивной подгруппы и унипотентного радикала. Мы доказываем, что тогда минимальность левого $H$-действия на $G / \Gamma$ влечет его строгую эргодичность. Попутно мы излагаем редукцию изучения конечных эргодических мер для любого действия $(G / \Gamma, H)$, где подгруппа $H \subset G$ связна и $\Gamma \subset G$ дискретна, к случаю абелевой подгруппы $H$.

Библиография: 19 названий.

Пусть $G$ - группа Ли и $D \subset G$-замкнутая подгруппа. $G$-инвариантную меру на $G / D$ (если она существует) будем называть мерой Хаара. Если мера Хаара на $G / D$ конечна, то говорят, что пространство $G / D$ имеет конечны й обвем. Если к тому же подгруппа $D \subset G$ дискретна, она называется решеткой в $G$.

Пусть $(G / D, H)$ обозначает левое действие связной подгрупшы $H$ группы Ли $G$ на однородном пространстве $G / D$ конечного объема. Мы будем изучать связь таких свойств этой динамической системы, как минимальность и строгая эргодичность. Напомним, что действие назьвается минимальным, если все его орбиты плотны в $G / D$ (иначе говоря, $\overline{H x}=G / D$ для любой точки $x \in G / D)$. Действие называется строго әргодичес$\kappa u м$, если $H$-инвариантная вероятностная мера на $G / D$ единственна (в таком случае она совпадает с нормированной мерой Хаара).

Предположим, что непрерьвное действие аменабельной групшы $A$ на компактном топологическом пространстве $X$ строго эргодично и единственная инвариантная мера положительна на открытых подмножествах в $X$. Хорошо известно, что такое действие минимально. Интересно, что в классе однородных действий $(G / D, H)$ это вьполняется для любых связных подгрупп $H \subset G$.

ЛЕмма 1. Пусть $G / D$ - однородное пространство конечного обвема $и H \subset G$ связная подгруппа. Тогда любое компактное Н-инвариантное подмнохество $M \subset G / D$ имеет H-инвариантную вероятностную меру. В частности, если пространство $G / D$ компактно, то строго әргодическое действие $(G / D, H)$ является минимальным.

Работа выполнена при поддержке Российского фонда фундаментальных исследований, грант № 97-01-00456. 
ДокАЗАтЕльство. Если подгруппа $H$ аменабельна, у нас нет проблем. Пусть теперь $H=S_{H} \cdot A_{H}$ - разложение на полупростую подгруппу $S_{H} \subset H$ без компактных множителей и нормальную аменабельную подгрупш $A_{H} \subset H$. Если $P_{H} \subset S_{H}-$ разрешимая параболическая подгруппа, то группа $B=P_{H} \cdot A_{H}$ аменабельна, поэтому $M$ имеет хотя бы одну $B$-инвариантную вероятностную меру $\mu$. По теореме Ратнер [1] любая $P_{H}$-инвариантная вероятностная мера является $S_{H}$-инвариантной. Следовательно, мера $\mu$ является $H$-инвариантной.

Заметим, что даже для однородных действий из строгой эргодичности, вообще говоря, не следует минимальность. А именно, Рагунатан (см. [2]) построил пример действия $(G / D, H)$ на некомпактном пространстве $G / D$ конечного объема, которое является строго эргодическим, но не минимальным (в этом примере $H$ является расщепимой разрешимой четырехмерной подгруппой в $G=\mathrm{SL}(3, \mathbb{R})$, а действие обладает замкнутой некомпактной орбитой $H x \subset G / D)$.

В работе [3] Фюрстенберг поставил проблему: верно ли, что минимальность однородного действия $(G / D, H)$ на однородном пространстве конечного объема влечет его строгую эргодичность? Напомним известные на сегодняшний день результаты в этом направлении, для чего введем несколько определений.

Пусть $\mathrm{Ad}: G \rightarrow \operatorname{Aut}(\mathfrak{g})$ - присоединенное представление связной вещественной группы Ли $G$ на своей алгебре Ли g. Напомним, что группа автоморфизмов $\operatorname{Aut}(\mathfrak{g})$ является вещественно-алгебраической матричной группой. Скажем, что элемент $g \in G$ является Ad-унипотентным (соответственно Ad-квазиунипотентным), если все собственные значения матрищы $\mathrm{Ad}_{g}$ равны 1 (соответственно лежат на единичной окружности в $\mathbb{C}$ ). Элемент $g \in G$ называется Ad-nолупростым (соответственно

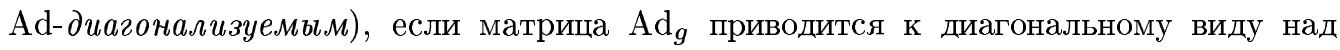
полем $\mathbb{C}$ (соответственно над $\mathbb{R})$.

Благодаря фундаментальным результатам Марины Ратнер (см. обзоры [1] и [4]) на данньй момент хорошо изучены действия $(G / D, H)$ для любой связной подгруппы $H$, порожденной Ad-унипотентными элементами. Дело в том, что все конечные $H$-инвариантные эргодические меры на $G / D$ имеют алгебраическое происхождение. А именно, любая такая мера совпадает с $F$-инвариантной мерой Хаара на некоторой замкнутой орбите $F x \subset G / D$ (при этом $H \subset F \subset G$ ). Аналогичное утверждение имеет место и для замыканий орбит действия $(G / D, H)$ при условии, что $G / D$ имеет конечный объем. Точнее, для любой точки $x \in G / D$ найдется связная замкнутая подгрупша $F \supset H$ такая, что $\overline{H x}=F x \subset G / D$, причем $F$-инвариантная мера Хаара на орбите $F x$ конечна. В частности, для таких действий минимальность и строгая эргодичность эквивалентны.

Используя результаты Ратнер и существование ограниченных орбит у любого однопараметрического однородного потока, установленное Клейнбоком и Маргулисом [5], автор в [6] доказал эквивалентность минимальности и строгой эргодичности для любой одномерной подгрупшы $H \subset G$.

Мозес и Вейсс [7] показали, что минимальность влечет строгую эргодичность при условии, что $G$ является $\mathbb{R}$-алгебраической полупростой групой, а $H$ - ее $\mathbb{R}$-алгебраической подгруппой.

Скажем, что связная подгруппа $H \subset G$ является Ad-расшепимой, если $\operatorname{Ad}(H)$ расшепляется в полупрямое произведение максимальной редуктивной подгруппы и унипотентного радикала (хорошо известно, что такое расщепление всегда имеет место, если подгруппа $\operatorname{Ad}(H) \subset \operatorname{Aut}(\mathfrak{g})$ алгебраична). Эквивалентное определение состоит в том, 
что $H=H_{s} H_{u}$, где $H_{u}$ - нормальная подгруппа в $H$, порожденная всеми Ad-унипотентными элементами, а подгруппа $H_{s}$ состоит из Ad-полупростых элементов.

Наша основная теорема усиливает результат [7].

Tеорема 2. Пусть Г-решетка в связной группе Ли $G$ и $H \subset G$ - еe Ad-pacщепимая связная подгруппа. Тогда из минимальности действия $(G / \Gamma, H)$ следует его строгая әргодичность.

В п. 1, 2 мы доказьваем несколько простых результатов о минимальности, строгой эргодичности и структуре однородных пространств конечного объема. В п. 3 формулируем результат в духе Мозеса [8], Маргулиса и Томанова [9], затем используем его для редукции изучения $H$-инвариантных мер на $G / \Gamma$, где $H$ - любая связная подгруппа в $G$, к случаю абелевой подгруппы $H$. Это позволит нам в п. 4 свести доказательство теоремы 2 к случаю $H=C \times H^{\prime}$, где $C$ - компактная подгруппа, а подгрушпа $\operatorname{Ad}\left(H^{\prime}\right)$ является $\mathbb{R}$-диагонализуемой. Затем в п. 5 мы показываем, что действие такой подгруппы $H$ может быть минимальным, лишш если $H^{\prime}$ является центральной подгруппой в $G$. В п. 6 завершаем наше доказательство.

1. Несколько общих замечаний о минимальности и строгой эргодичности. В этом пунктемы излагаем несколькопростых результатов, которыепотребуются в дальнейшем. Пусть $X$ - топологическоепространство, снабженное непрерывным действием групшы Ли $H$. Напомним, что такое действие называется минимальным, если $X$ не содержит собственных замкнутых $H$-инвариантньх подмножеств (т.е. все $H$-орбиты плотны в $X$ ). Следуя [10], действие $H$ на $X$ назовем поточечно-минимальным, если $X$ является дизъюнктным объединением замкнутых $H$-минимальных подмножеств. Иначе говоря, замыкания любых двух $H$-орбит в $X$ либо совпадают, либо не пересекаются.

ЛЕмма 1.1 [10]. Пусть $H$ - группа Ли, непрерывно действующая на топологическом пространстве $X$. Если $F \subset H$ - замкнутая нормальная подгруппа такая, что $H / F$ - компакт, то действие $F$ на $X$ поточечно-минимально.

СлЕДСТВИЕ 1.2 [7]. Пусть $G$ - связная әруппа Ли и действие $(G / D, H)$ минимально. Предположим, что $D^{\prime} \subset D$ - нормальная подгруппа конечного индекса. Тогда действие $\left(G / D^{\prime}, H\right)$ минимально.

ЛЕмма 1.3. Пусть $G$ - связная группа Ли $и D, H \subset G-$ ее замкнутые подгрупnы. Пусть также $C \subset H$ - компактная подгруппа и $G^{\prime} \supset D$ - замкнутая подгруппа такая, что $G=C G^{\prime}$ (т.е. каждый әлемент $g \in G$ может быть записан в виде произведения $g=c g^{\prime}$, где $\left.c \in C, g^{\prime} \in G^{\prime}\right)$. Предположим, что $H^{\prime} \subset H \cap G^{\prime}-$ нормальная подгруппа в $H$. Тогда из строгой эргодичности действия $\left(G^{\prime} / D, H^{\prime}\right)$ следует строгая әргодичность действия $(G / D, H)$.

ДокАЗАТЕЛЬСтво. Пусть $\mu$ обозначает $H$-инвариантную вероятностную меру на $G / D$. Разложим ее в условные меры по отношению к расслоению пространства $G / D$ на замкнутые подпространства $g G^{\prime} D \subset G / D$. Все эти подпространства $H^{\prime}$-инвариантны, поэтому все условные меры также $H^{\prime}$-инвариантны. Но $H^{\prime}$-действие на каждом слое $g G^{\prime} D$ гладко изоморфно действию $\left(G^{\prime} / D, H^{\prime}\right)$, поэтому все условные меры являются мерами Хаара (на соответствующем подпространстве). Стало быть, $\mu$ есть полупрямое произведение $H$-инвариантной меры на $G / G^{\prime}$ и меры Хаара на $G^{\prime} / D$ посредством расслоения $G / D$ над $G / G^{\prime}$ со слоями, изоморфными $G^{\prime} / D$ (см. [11]). 
Осталось заметить, что $H$-инвариантная мера на $G / G^{\prime}=C / C \cap G^{\prime}$ является мерой Xaapa.

ЛЕмма 1.4 [12]. Пусть $G / D$ - пространство конечного обгема, причем груп$n$ пь $D$ и $G$ унимодулярны. Тогда если подгруппа $H \subset G$ нормальна и унимодулярна и $G=\overline{H D}$, то действие $(G / D, H)$ строго әргодично.

2. Еще раз об однородных пространствах конечного объема. Скажем, что замкнутая подгруппа $D \subset G$ является квазирешеткой, если пространство $G / D$ имеет конечньй объем и $D$ не содержит нетривиальных связных нормальных в $G$ подгрупп. Обозначим через $R$ радикал связной групы Ли $G$, через $L$ подгрупп Леви. Запись $L=S K$ будет обозначать почти прямое разложение $L$ на нормальную подгрупу $S$ без компактных множителей и компактную нормальную подгрупу $K$. Пусть, наконец, $\operatorname{Int}_{F} H=\left\{f H f^{-1}, f \in F\right\}$. Напомним следующий результат.

Лемма 2.1 [13], [14]. Пусть $G / D$ - пространство конечного обгема $u G=\overline{S R D .}$ Tогда произведение $R D$ замкнуто. Ecли, кроме того, $D$ является квазирешеткой, то $D_{0} \subset R$ и $D \cap R$ является квазирешеткой в $R$.

Следуюший результат хорошо известен, если $D$ является решеткой в $G$, но, по-видимому, не был доказан в общем случае.

ЛЕмма 2.2. Пусть пространство G/D имеет конечный обтем. Тогда произведение $K R D$ замкнуто. Если, кроме того, $D$ является квазирешеткой, то $D_{0} \subset K R$ и $D \cap K R$ является квазирешеткой в $K R$.

ДокАЗАТЕЛЬСТво. Без ограничения общности мы можем предположить, что $D$ является квазирешеткой и группа $G$ односвязна. В модификации Дани теоремы плотности Бореля [15] утверждается, что алгебраическая оболочка подгрупшы $\operatorname{Ad}(D) \subset \operatorname{Aut}(\mathfrak{g})$ содержит $\operatorname{Ad}(S N)$, где $N \subset R$ обозначает нильрадикал. Отсюда мы выводим, что группа $D_{0}$ аменабельна, т.е. она не содержит некомпактных простьх подгрупп. В самом деле, если $S_{D} \subset S \cap D_{0}$ - такая подгруппа, то $\operatorname{Int}_{D_{0}} S_{D} \subset D_{0}$, поэтому $\operatorname{Int}_{S N} S_{D} \subset D_{0}$. С другой стороны, $K$ коммутирует с $S_{D}$, поэтому $\operatorname{Int}_{G} S_{D} \subset D_{0}$. Но поскольку $D$ является квазирешеткой, подгруппа $S_{D}$ должна быть тривиальной.

Пусть теперь $p_{K}: G \rightarrow K-$ проекция и $\left(\overline{p_{K}(D)}\right)_{0}=K_{1} T_{1} \subset K$ - почти прямое произведение полупростой группы Ли $K_{1}$ и тора $T_{1}$. Определим $G^{\prime}=\left(S \times K_{1}\right)\left(T_{1} R\right)$ и $D^{\prime}=D \cap G^{\prime}$. Тогда $D^{\prime}$ - подгруппа конечного индекса в $D$, а пространство $G^{\prime} / D^{\prime}$ имеет конечньй объем. Заметим, что группа $R^{\prime}=T_{1} \cdot R$ является радикалом в $G^{\prime}$, а произведение $S R^{\prime} D^{\prime}$ плотно в $G^{\prime}$. По лемме 2.1 произведение $R^{\prime} D^{\prime}$ замкнуто (поэтому пространство $R^{\prime} / D \cap R^{\prime}$ компактно). Следовательно, произведение $K R D$ также замкнуто. Поскольку група $D_{0}$ аменабельна, из теоремы плотности Бореля [11] следует, что проекция $D$ на $S$ является решеткой в $S$.

Наконец, если $W \subset D_{0}$ - максимальная среди связных нормальных в $K R$ подгрупп,

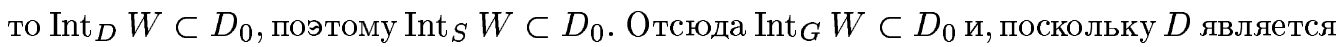
квазирешеткой в $G$, подгрупша $W$ должна быть тривиальной.

В ходе доказательства мы установили следующее утверждение.

Лемма 2.3. Пусть пространство $G / D$ имеет конечный облем. Тогда существует тор $T \subset K$ такой, что пространство $T R / D \cap T R$ компактно. 
Напомним, что этот результат (полученный независимо многими авторами в середине 80-х годов) доказывает гипотезу [16] о том, что любоепространство конечного объема допустимо.

3. Основная техническая теорема. Пусть подгруппа $H \subset G$ связна, а $\Gamma \subset G$ дискретна. Мы изложим редукцию изучения $H$-инвариантных вероятностных мер на $G / \Gamma$ к случаю, когда подгруппа $H$ абелева. Эта редукция играет ключевую роль в доказательстве главного результата.

Пусть $H_{u} \subset H$ обозначает связную нормальную подгруппу в $H$, порожденную всеми Ad-унипотентными однопараметрическими подгруппами. Согласно Ратнер (см. [1]) все $H_{u}$-инвариантные эргодические вероятностные меры являются мерами Хаара на однородных подпространствах в $G / D$.

Скажем, что подгруппа $H$ имеет Ad-треугольный тuп, если $H$ порождена элементами $h \in H$ такими, что все собственные значения оператора $\mathrm{Ad}_{h}$ на алгебре Ли $\mathfrak{g}$ чисто вещественные. Легко видеть, что следующие условия эквивалентны:

1) $H$ имеет Аd-треугольньй тип;

2) максимальная редуктивная подгруппа в алгебраической оболочке подгруппы $\operatorname{Ad}(H) \subset \operatorname{Aut}(\mathfrak{g})$ не имеет компактных нормальных подгрупп;

3) если $H=L_{H} R_{H}$ - разложение Леви, то подгруппа Леви $L_{H}$ не имеет компактных множителей, a $\operatorname{Ad}\left(R_{H}\right)$ сопряжена подгрупше в $\operatorname{Aut}(\mathfrak{g})$, состоящей из верхнетреугольньг матриц.

Ясно, что для такой $H \operatorname{Ad}[H, H]=[\operatorname{Ad}(H), \operatorname{Ad}(H)] \subset \operatorname{Ad}\left(H_{u}\right)$, поэтому факторгруппа $H / H_{u}$ абелева. Следующая теорема сводит изучение $H$-инвариантных вероятностных мер для любой подгруппы $H$, имеющей Ad-треугольньй тип, к случаю абелевой подгруппы $H$. Основная ее часть неявно содержится в работах Мозеса $[8$, доказательство теоремы 1] и Маргулиса и Томанова [9, основная теорема и ее доказательство для вещественньх групп Ли] (в обеих работах предполагалось, что $H$ порождена Ad-унипотентными и Ad-диагонализуемыми элементами; однако доказательство проходит для любой подгруппы Ad-треугольного типа); близкая формулировка содержится в работе Мозеса и Вейсса [7].

Tеорема 3.1. Пусть Г - дискретная подгруппа в $G, H \subset G$ - связная подгруппа Ad-треугольного типа и $\mu-H$-инвариантная эргодическая вероятностная мера на $G / \Gamma$. Тогда найдется связная замкнутая подгруппа $L \subset G$ такая, что н-почти

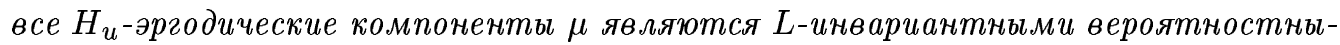
ми мерами на замкнутых L-орбитах в $G / \Gamma$. Пусть теперь

$P=S N_{G}(L)=\left\{p \in G:\right.$ сопря⿻ение $g \rightarrow \operatorname{pgp}^{-1}$ сохраняет $L$ и меру Хаара на ней $\}$.

Тогда $H \subset P$ и найдется точка $x \in G / \Gamma$ такая, что орбита $P x \subset G / \Gamma$ замкнута $u \mu(P x)=1$.

Из теоремы 3.1 следует, что однородное пространство $P x$ является $H$-инвариантным и $\mu(P x)=1$. Поскольку группа $L$ нормальна в $P$, то все $L$-орбиты в $P x$ замкнуты и образуют расслоение над однородным $P / L$-пространством. Заметим, что $H_{u} \subset L$, мера $\mu$ является $L$-инвариантной и факторгрупа $H / H_{u}$ абелева. Таким образом, изучение $H$-инвариантных вероятностных мер для подгрупп Ad-треугольного типа сводится к случаю, когда подгруппа $H$ абелева. 
Если $H \subset G$ - произвольная связная подгрупа, то иногда она расщепляется в полупрямое произведение $H=C \cdot H^{\prime}$ компактной подгрупшы $C$ и нормальной подгрупшы $H^{\prime}$, имеющей $\mathrm{Ad}$-треугольньй тип. Это позволяет описьвать $H$-инвариантные меры, используя знание $H^{\prime}$-инвариантных мер.

В общем случае можно действовать следующим образом. Без ограничения общности будем считать, что группа $G$ связна и односвязна. Предположим вначале, что подгруппа $H$ разрешима. Рассмотрим алгебраическую оболочку $\mathscr{A}(\operatorname{Ad}(H))$ в вещественно-алгебраической групше Ли $\operatorname{Aut}(\mathfrak{g})$. Тогда $\mathscr{A}(\operatorname{Ad}(H))=T \cdot M$, где $T$-компактный тор и $M-$ нормальная триангуляризуемая подгрупа. Пусть $\widetilde{G}=T \cdot G$ и $\widetilde{H}=T \cdot H$. Легко видеть, что $\widetilde{H}=T \cdot H^{\prime}$, где $H^{\prime} \subset \widetilde{H}$ - нормальная подгруппа Ad-треугольного типа. Заметим, что $H$-инвариантные меры на $G / \Gamma$ находятся во взаимно однозначном соответствии с $\widetilde{H}$-инвариантньми мерами на $\widetilde{G} / \Gamma$. В частности, действие $(G / \Gamma, H)$ строго эргодично тогда и только тогда, когда действие $(\widetilde{G} / \Gamma, \widetilde{H})$ таково. То же относится и к орбитам. В частности, действие $(G / \Gamma, H)$ минимально в том и только том случае, когда действие $(\widetilde{G} / \Gamma, \widetilde{H})$ минимально.

Для общей подгрупшы $H=L_{H} R_{H}$ проделаем описанную вьше процедуру с ее радикалом $R_{H}$ и заметим, что тор $T \operatorname{Aut}(\mathfrak{g})$ можно выбрать коммутирующим с $L_{H}=K_{H} S_{H}$. Более того, $T \cdot R_{H}=T \cdot R_{H}^{\prime}$, где $R_{H}^{\prime} \subset \widetilde{H}=T \cdot H$-нормальная подгруппа Аd-треугольного типа в $\widetilde{G}=T \cdot G$. Тогда $\widetilde{H}=C H^{\prime}$, где $C=K_{H} \times T$ и $H^{\prime}=S_{H} R_{H}^{\prime} \subset \widetilde{H}$ - нормальная подгруппа Ad-треугольного типа. В этом случае снова изучение инвариантных мер (и орбит) для действий $(G / \Gamma, H)$ и $(\widetilde{G} / \Gamma, \widetilde{H})$ эквивалентны.

4. Редукция к случаю, когда $H$ состоит из $\mathrm{Ad-полупростых} \mathrm{элементов.}$ Скажем, что связная подгрупша $H \subset G$ является Ad-uзотропной, если $H=H_{s} H_{u}$, где подгруппа $\operatorname{Ad}\left(H_{s}\right) \subset \operatorname{Aut}(\mathfrak{g})$ является $\mathbb{R}$-диагонализуемой. Легко видеть, что $H$ является Ad-изотропной тогда и только тогда, когда она одновременно Ad-расщепима и имеет $\mathrm{Ad}$-треугольный тип.

Для доказательства теоремы 2 мы применим результаты п. 3. Пусть $(G / \Gamma, H)$ - минимальное действие Ad-расщепимой подгруппы $H$. Как уже отмечалось в п. 3 , без ограничения общности можно считать, что $H=C H^{\prime}$, где подгруппа $C$ компактна и $H^{\prime}-$ нормальная Ad-изотропная подгруппа в $H$. Пусть $\mu-H$-инвариантная вероятностная мера на $G / \Gamma$. Применив теорему 3.1 к любой из ее $H^{\prime}$-эргодических компонент, мы найдем подгрупу $L \subset G$ и точку $x \in G / \Gamma$ такие, что $\overline{H_{u} x}=L x$ и орбита $P x \subset G / \Gamma$ замкнута, где $P=S N_{G}(L) \supset H^{\prime}$. Поскольку $H \subset C P$ и $H$-действие минимально, мы выводим равенство $G=C P$ (действительно, $G$ покрывается счетным семейством $C P \Gamma$ замкнутых многообразий, поэтому $C P$ замкнуто и открыто в $G$ ).

Так как $P$ нормализует $L$, а $C$ нормализует $H_{u}$, любая сопряженная к $H_{u}$ подгруппа лежит внутри $L$. Следовательно, $\overline{H_{u} x}=\overline{U x}=L x$, где $U \subset G$ - наименьшая нормальная подгруппа, содержащая $H_{u}$. Это означает, что $H^{\prime}$-эргодическая компонента $\mu$, сосредоточенная на $P x$, является мерой Хаара на каждом слое $\bar{U} y, y \in P x$. Аналогично мы выводим, что $\mu$ является мерой Хаара на всех слоях $\overline{U y}, y \in G / \Gamma$. Точнее, $\mu$ есть полупрямое произведение посредством однородного расслоения $G / \Gamma \rightarrow G / \overline{U \Gamma}$ меры $\mu_{1}$ на пространстве $G / \overline{U \Gamma}$ и меры Хаара на $\overline{U \Gamma} / \Gamma$.

Пусть $W \subset(\overline{U \Gamma})_{0}$ - наибольшая связная нормальная подгруппа в $G, G_{1}=G / W$, $\Gamma_{1}=\overline{U \Gamma} / W, H_{1}=H W / W$. Тогда действие $\left(G_{1} / \Gamma_{1}, H_{1}\right)$ минимально и $\mu_{1}$ есть $H_{1}$-инвариантная вероятностная мера на пространстве конечного объема $G_{1} / \Gamma_{1}$. Поскольку $H_{u} \subset U \subset W$, подгруппа $H_{1}$ состоит из Ad-полупростых элементов. Ясно, что до- 
статочно доказать $G_{1}$-инвариантность меры $\mu_{1}$. Относительно $\Gamma_{1}$ мы знаем, что это квазирешетка в $G_{1}$ (но не обязательно решетка). Также заметим, что $H_{1}$ есть коммутативное произведение своей максимальной компактной подгруппы и абелевой подгрупш, состоящей из Ad-диагонализуемых элементов.

5. Разрешимый случай. Пусть $H \subset G$ - связная подгрупша и $U \subset G$ - неустойчивая нормальная подәруппа по отношению к $H$ (см. [16], [17]). По определению $U$ - наименьшая нормальная подгруппа в $G$ такая, что образ $H$ в $G / U$ состоит из $\mathrm{Ad}$-квазиунипотентных элементов. Если $\operatorname{Ad}(H)$ является $\mathbb{R}$-диагонализуемой, то образ $H$ в $G / U$ является центральной подгруппой и действие $(G / D, H)$ эргодично в том и только том случае, когда $G=\overline{H U D}$.

Лемма 5.1. Пусть $G$ - связная разрешимая группа Ли, $H \subset G-$ связная абеле-

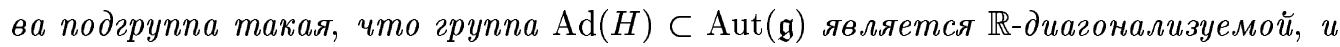
$(G / D, H)$ - поточечно-минимальное действие на компактном разрешимом многообразии. Тогда $U \subset D_{0}$. Другими словами, $H$ является иентральной подгруппой в $G$ ( и потому сама группа $G$ абелева) при условии, что $D$ - квазирешетка в $G$.

ДоКАЗАТЕЛЬСТво. Без ограничения общности мы можем считать, что действие $(G / D, H)$ эргодично. В самом деле, определим $G^{\prime}=(\overline{H U D})_{0}$. Тогда $G^{\prime}$ является подгруппой в $G$, а $D^{\prime}=D \cap G^{\prime}$ имеет конечный индекс в $D$. Действие $\left(G^{\prime} / D^{\prime}, H\right)$ эргодично и по следствию 1.2 поточечно-минимально. Осталось заметить, что $U \subset G^{\prime}$ и $D_{0}^{\prime}=D_{0}$.

Итак, мы можем считать действие $(G / D, H)$ эргодичным (и потому минимальным), группу $G$ связной и односвязной, а $D$ квазирешеткой в $G$. Теперь воспользуемся $\kappa о н с m$ рукиией полупростого расщепления группы $G$ (см. [16], [17]). Пусть $\mathscr{A}(\operatorname{Ad}(G))=$ $A \cdot M \subset \operatorname{Aut}(\mathfrak{g})$ - разложение алгебраической оболочки $\operatorname{Ad}(G)$ в полупрямое произведение абелевой редуктивной подгруппы $A$ и унипотентного радикала $M$. Определим $A(G)=A \cdot G$. Тогда $A(G)=A \cdot W$, где $W$ - нильрадикал в $A(G)$, назьваемьй нильтенью $G$. Группа $A(G)$ допускает структуру вещественно-алгебраической групы, в которой $A$ является максимальной редуктивной подгруппой, а $W$ - унипотентным радикалом (ясно, что группа $G$ нормальна и плотна по Зарисскому в $A(G)$ ). Заметим, что все Ad-унипотентные элементы в $G$ являются унипотентными в $A(G)$ (поэтому лежат в $W$ ). Каждый Ad-полупростой элемент $g \in G$ после подходящего сопряжения лежит в $A \times Z$, где $Z \subset W$ обозначает центр группы $A(G)$.

Пусть $p: A(G)=A \cdot W \rightarrow A$-естественная проекция вдоль $W$. Если $D$-квазирешетка в $G$, то подгруппа $T_{G}=p(G) \subset A$ замкнута в евклидовой топологии и $T_{D}=p(D)-$ решетка в $T_{G}$ (усиленная теорема Мостова из [17]).

Известно (см. [17]), что для любой равномерной подгруппы $D \subset G$ ее алгебраическая оболочка $\mathscr{A}(D) \subset A(G)$ нормальна и равномерна. В частности, $\mathscr{A}(D)$ содержит все унипотентные и $\mathbb{R}$-диагонализуемые элементы из $A(G)$. Следовательно, $H \subset \mathscr{A}(D)$ и ввиду эргодичности $\mathscr{A}(D)=A(G)$, т.е. $D$ плотна по Зарисскому в $A(G)$. В частности, $D_{0}$ нормальна в $G$. Поэтому $D$ - (плотная по Зарисскому) решетка в $G$.

Так как $H$ - абелева группа из Ad-полупростых элементов, она сопряжена подгруппе в $A \times Z$. Поэтому мы можем считать, что $A$ коммутирует с $H$. Докажем, что группа $G$ абелева. Для этого достаточно рассмотреть случай, когда нильтень $W$ абелева. В самом деле, произведение $D[W, W]$ замкнуто, и мы можем заменить $G$ на $G^{\prime}=G /[W, W]$, а $D$ на $D^{\prime}=D[W, W] /[W, W]$. Если мы докажем, что группа $G^{\prime}$ абелева, это будет означать, что $[A(G), A(G)]=\mathscr{A}([G, G]) \subset[W, W]$, поэтому группа $A$ тривиальна, т.е. 
группа $G$ нильпотентна. Но для нильпотентной групшы $G$ нет проблем: $H$ лежит в центpe $G$ и поэтому действует минимально на $G / D$, лишь если групша $G$ абелева.

Итак, мы можем предположить, что нильтень $W$ является векторной группой. Тогда $\widetilde{D}=T_{D} D$ - плотная по Зарисскому дискретная подгрупша в $A(G)$, которая пересекает $W$ по решетке. Снова мы можем считать, что $H \subset A \times Z$. Так как $\mathscr{A}(\widetilde{D})=A(G)$, произведение $Z \widetilde{D}$ замкнуто, значит произведение $(A \times Z) \widetilde{D}$ также замкнуто. Из минимальности следует, что $G \subset A \times Z$. Но это означает, что группа $G$ абелева (и поэтому $A(G)=G=W=Z)$. Лемма доказана.

6. Доказательство теоремы 2. Итак, мы имеем минимальное действие $(G / D, H)$, где $H=C \times H^{\prime}$. Здесь $C$-компактная подгруппа, а группа $\operatorname{Ad}\left(H^{\prime}\right)$ является $\mathbb{R}$-диагонализуемой. Используя лемму 2.2 , можно легко показать, что в данной ситуации $G=K \cdot R$ (см. обозначения п. 2). В самом деле, пусть $p: G \rightarrow S$ - проекция. Тогда $p(D) \subset S$ является решеткой. Пусть $S^{\prime} \subset S$ - нормальная подгруппа такая, что решетка $p(D) \cap S^{\prime}$ неприводима, и $p^{\prime}: G \rightarrow S^{\prime}-$ соответствующая проекция. Тогда согласно критерию эргодичности Мура [18] действие $\left(S^{\prime} / p^{\prime}(D), p^{\prime}\left(H^{\prime}\right)\right)$ эргодично при условии, что проекция $p^{\prime}\left(H^{\prime}\right)$ нетривиальна. С другой стороны, она лежит в картановской подгруппе группы $S^{\prime}$. Но действие картановской подгрупшы на $S^{\prime} / p^{\prime}(D)$ всегда обладает инвариантньм тором (Мостов, Прасад и Рагунатан [19]), поэтому не может быть поточечно-минимальным. Следовательно, проекция $p\left(H^{\prime}\right)$ тривиальна и потому проекция $p(H)$ компактна. Но компактная подгруппа может действовать минимально на $S / p(D)$, лишь если группа $S$ тривиальна.

Таким образом, $G=K \cdot R$ и $H=C \times H^{\prime}$, где группа $\operatorname{Ad}\left(H^{\prime}\right)$ является $\mathbb{R}$-диагонализуемой; в частности, $H^{\prime} \subset R$. Используя лемму 2.3 , найдем тор $T \subset K$ такой, что пространство $T R / D \cap T R$ компактно. По лемме 1.1 действие $\left(T R / D \cap T R, H^{\prime}\right)$ поточечно-минимально, а из леммы 5.1 следует, что связная компонента группы $D \cap T R$ содержит неустойчивую нормальную подгруппу $U$ для групшы $H^{\prime}$. Но $D$ - квазирешетка в $G$, поэтому подгрупша $H^{\prime}$ центральна в $G$.

Определим теперь $G^{\prime}=\left(\overline{H^{\prime} D}\right)_{0}$. Поскольку действие $(G / D, H)$ минимально, имеем $G=C G^{\prime}$. С другой стороны, по лемме 1.4 действие $\left(G^{\prime} / D \cap G^{\prime}, H^{\prime}\right)$ строго эргодично. Осталось применить лемму 1.3 .

ДоБАВлЕниЕ. После того, как эта работа была написана, автор получил препринт Томанова "On unique ergodicity for subgroup actions on homogeneous spaces", в котором независимо доказьвается по существу тот же результат, что и в теореме 2 . Автор благодарен Г. Томанову за обсуждение наших работ.

\section{СПИСОК ЦИТИРОВАННОЙ ЛИТЕРАТУРЫ}

[1] Ratner M. Interactions between ergodic theory, Lie groups, and number theory // Proc. of the ICM. Zurich (Switzerland), 1994. Zurich, 1995. P. 157-182.

[2] Weiss B. Finite-dimensional representations and subgroup actions on homogeneous spaces // Israel J. Math. 1998. V. 106. P. 189-207.

[3] Furstenberg H. The unique ergodicity of the horocycle flow // Recent Advances in Topological Dynamics. Lecture Notes in Math. V. 318. Berlin: Springer, 1972. P. 95-115.

[4] Старков А.Н. Новый прогресс в теории однородных потоков // УМН. 1997. Т. 52. C. 87-192.

[5] Kleinbock D. Y., Margulis G. A. Bounded orbits of non-quasiunipotent flows on homogeneous spaces // Amer. Math. Soc. Transl. Ser. 2. 1996. V. 171. P. 141-171. 
[6] Starkov A. N. Minimal sets of homogeneous flows // Ergodic Theory Dynamical Systems. 1995. V. 15. P. 361-377.

[7] Mozes S., Weiss B. Minimality and unique ergodicity for subgroup actions // Ann. Inst. Fourier (Grenoble). 1998. V. 48. № 5. P. 1533-1541.

[8] Mozes S. Epimorphic subgroups and invariant measures // Ergodic Theory Dynamical Systems. 1995. V. 15. P. 1207-1210.

[9] Margulis G. A., Tomanov G. Measure rigidity for almost linear groups and its applications // J. Anal. Math. 1996. V. 69. P. 25-54.

[10] Gottschalk W. H., Hedlund G. A. Topological Dynamics. Amer. Math. Soc. Colloq. Publ. V. 36. Providence (R.I.), 1955.

[11] Рагунатан М. С. Дискретные подгруппы групп Ли. М.: Мир, 1977.

[12] Margulis G. A., Tomanov G. Invariant measures for actions of unipotent groups over local fields on homogeneous spaces // Invent. Math. 1994. V. 116. P. 347-392.

[13] Старков А. Н. О критерии эргодичности $G$-индуцированных потоков // УМН. 1987. Т. 42. C. $233-234$.

[14] Witte D. Zero-entropy affine maps on homogeneous spaces // Amer. J. Math. 1987. V. 109. P. 927-961.

[15] Dani S. G. A simple proof of Borel's density theorem // Math. Z. 1980. V. 174. P. 81-94.

[16] Brezin J., Moore C. C. Flows on homogeneous spaces: a new look // Amer. J. Math. 1981. V. 103. № 3. P. 571-613.

[17] Auslander L. An exposition of the structure of solvmanifolds // Bull. Amer. Math. Soc. 1973. V. 79. P. 227-261.

[18] Moore C. C. Ergodicity of flows on homogeneous spaces // Amer. J. Math. 1966. V. 88. P. 154-178.

[19] Prasad G., Raghunathan M.S. Cartan subgroups and lattices in semisimple groups // Ann. of Math. (2). 1972. V. 96. P. 296-317.

Русско-датский институт эффективности энергии, г. Москва

Поступило

(Russian-Danish Institute of Energy-efficiency)

25.12 .98

E-mail: alex@rdiees.msk.ru 